\title{
Focused Ion Beam Assisted Interface Detection for Fabricating Functional Plasmonic Nanostructures
}

\author{
Houxiao Wang, ${ }^{1,2,3}$ Wei Zhou, ${ }^{2,3}$ and Er Ping $\mathrm{Li}^{3}$ \\ ${ }^{1}$ School of Mechanical Engineering, Jiangsu University, 301 Xuefu Road, Zhenjiang 212013, Jiangsu, China \\ ${ }^{2}$ School of Mechanical and Aerospace Engineering, Nanyang Technological University, 50 Nanyang Avenue, Singapore 639798 \\ ${ }^{3}$ Advanced Photonics and Plasmonics Division, A STAR Institute of High Performance Computing, \\ 1 Fusionopolis Way, No. 16-16 Connexis, Singapore 138632
}

Correspondence should be addressed to Houxiao Wang; wanghx@ujs.edu.cn and Wei Zhou; mwzhou@ntu.edu.sg

Received 24 January 2015; Accepted 8 April 2015

Academic Editor: Zhida Xu

Copyright (C) 2015 Houxiao Wang et al. This is an open access article distributed under the Creative Commons Attribution License, which permits unrestricted use, distribution, and reproduction in any medium, provided the original work is properly cited.

\begin{abstract}
Plasmonic nanoscale devices/structures have gained more attention from researchers due to their promising functions and/or applications. One important technical focus on this rapidly growing optical device technology is how to precisely control and fabricate nanostructures for different functions or applications (i.e., patterning end points should locate at/near the interface while fabricating these plasmonic nanostructures), which needs a systematic methodology for nanoscale machining, patterning, and fabrication when using the versatile nanoprecision tool focused ion beam (FIB), that is, the FIB-assisted interface detection for fabricating functional plasmonic nanostructures. Accordingly, in this work, the FIB-assisted interface detection was proposed and then successfully carried out using the sample-absorbed current as the detection signal, and the real-time patterning depth control for plasmonic structure fabrication was achieved via controlling machining time. Besides, quantitative models for the sampleabsorbed currents and the ion beam current were also established. In addition, some nanostructures for localized surface plasmon resonance biosensing applications were developed based on the proposed interface detection methodology for FIB nanofabrication of functional plasmonic nanostructures. It was shown that the achieved methodology can be conveniently used for real-time control and precise fabrication of different functional plasmonic nanostructures with different geometries and dimensions.
\end{abstract}

\section{Introduction}

Some methods and systems, for example, nanoimprinting lithography $[1,2]$, electron beam lithography [3-5], etching (reactive ion etching and dry etching) [6-10], femtosecond laser [11], interference lithography [12], and focused ion beam (FIB) [13-16], have been reported to develop optical structures or devices (e.g., specific functional plasmonic nanostructures). Compared with these reported methods and systems, despite the potential drawbacks of surface damage caused by high-energy ion beam irradiation and relatively limited processing speed for large-area high-density structural patterns $[6,13]$, the FIB has its strength in onestep maskless simpler, more flexible, and better-controlled nanoprecision machining, patterning, and fabrication especially for submicron, nanoscale, or subwavelength various functional samples or structures via ion beam induced milling, etching, and deposition due to its advantages of large depth of focus, high resolution, patterning flexibility, and direct-writing capability [6, 13-17]. The FIB system has been widely used in the fields of semiconductor, microand nanoelectronics including integrated-circuit modification and failure analysis, mask repairing, sample preparation for transmission electron microscopy (TEM), biomedicine, and micromachining [18]. Recently, the FIB nanoprecision machining, patterning, and fabrication for specific nanoscale applications have attracted more interest to realize more diversity for nanoconstruction $[6,13-16,19]$.

Some metals (e.g., $\mathrm{Au}, \mathrm{Ag}$, and $\mathrm{Cu}$ ) can be deposited on the dielectric substrate (e.g., $\mathrm{SiO}_{2}$ ) to develop different functional plasmonic structures/devices, which have gained more and more attention from the researchers due to their promising functions and/or applications because the interface between metal coating and dielectric substrate may 
support surface plasmon polaritons (SPPs) [20-22]. Accordingly, the patterning end points should locate at or near the interface while fabricating/developing these plasmonic structures/devices, which requires the interface detection to realize real-time patterning control and precise structure fabrication. However, this rapidly growing optical device technology requires a systematic methodology to precisely control and fabricate the designed nanoscale devices or structures for various industrial and commercial applications even while using the advanced nanoprecision tool FIB. Thus, it is necessary to carry out a systematic study on the FIBassisted interface detection for nanofabrication of different functional plasmonic nanostructures. Moreover, the rapidly growing applications of FIB in the field of nanomachining, nanopatterning, or nanofabrication especially for the plasmonic device development also require the capability to reliably detect the interfaces between different material layered structures, that is, the end point detection (EPD) for the accurate transition signal from one layer to the next $[23,24]$. The EPD technique allowed interaction of ions with sample surface to be monitored through a plot of stage grounding current as a function of ion milling time [25].

During ion milling, the sample-absorbed current, which depends on the beam current and the material-dependent number of the generated secondary electrons and ions, flows to the ground if the specimen is grounded, and it linearly increases with the beam current for the same material and changes with materials for the same beam current used [23, 24]. Because different materials have different characteristic secondary ion yields, it is possible to distinguish the interfaces between different material layers [26]. Thus, various materials can be identified while milling the multilayered structure made up of different materials via monitoring the current flow to the ground and then providing indications of the end points $[23,24,26-30]$.

For the plasmonic or multilayered nanostructures, it is important to know whether nanopatterns are formed at or near the interface between different material layers [27]. The end point monitor (EPM) in the FIB system may solve this problem by measuring the sample-absorbed current during the machining process, and the sudden current alteration indicates machining transition from one material layer to another [23, 24, 26-30]. The EPM-detected current signal alters as various material layers for a complex specimen are exposed for removal via the ion beam, and it can be real-time monitored (sample-absorbed current may be continuously monitored during FIB machining or fabrication process through connecting the sample to an electrometer amplifier, and the endpoint detection can be achieved via the detected current signal curve based on the real-time monitor) [23-25]. The real-time EPM current signal curve presents the sampleabsorbed current as a function of the machining time [23].

Among the functional plasmonic nanoscale structures or devices, the plasmonic nanobiosensors based on the localized surface plasmon resonance (LSPR) have become more and more important/interesting resulting from their promising functions and wide applications. The LSPR is sensitive to geometric parameters [31]. It is reported that

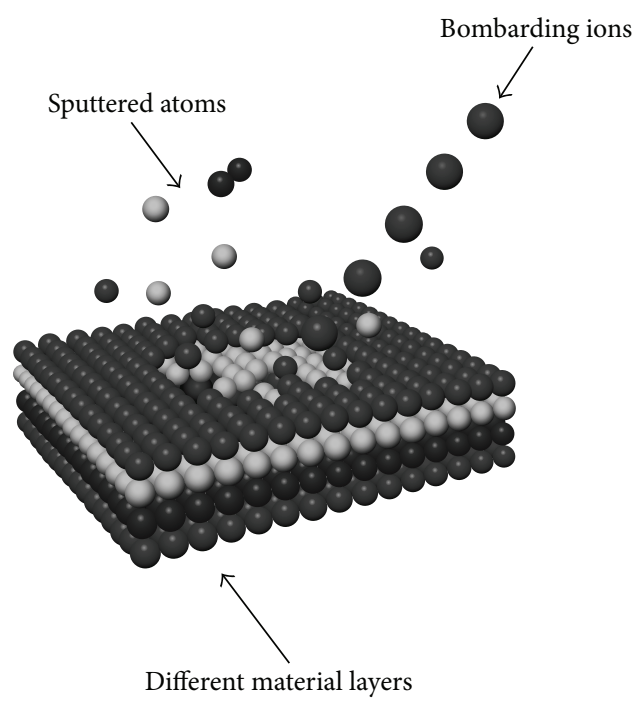

FIGURE 1: Schematic process for focused ion beam nanomachining.

many similar plasmonic nanohole/particle structures (e.g., the rhombic hybrid Au-Ag nanostructures [31], the nanohole array on a Au-deposited glass substrate [32], the Au/Ag nanoparticles [19], the square nanohole array [33], and nanostars [34]) have been applied for LSPR biosensing (e.g., a plasmonic square nanohole array can be used for the realtime sensing/monitoring of protein-protein specific binding interaction using a LSPR nanohole sensor integrated with microfluidic delivery, and the sensitivity of this nanohole sensor depended on the periodicity of the array and the order of the surface plasmon resonance modes) [19, 31-35], and they have been applied for sensing of localized transmission/reflection field intensity distribution [32], refractive index (e.g., sensing of the analytes of glucose water solutions with refractive indexes ranging from 1 to 1.38 ) [31, 34], and optical spectra (e.g., scattering spectra) $[33,34]$, and so forth.

So far, few literatures regarding investigation for the interface between different materials during FIB machining or fabrication process have been found, without mentioning the investigation of the interface detection between different materials during FIB machining for nanofabricating the functional plasmonic nanostructures. Different from the previous work regarding the interface detection using the EPD technique, this work has proposed and achieved a systematic methodology and detailed analysis regarding the interface detection for FIB nanofabrication of functional plasmonic nanostructures for biosensing applications through real-time patterning control using the EPM.

\section{Materials and Methods}

The specimen used consists of a quartz substrate and a very thin silver film layer deposited on the substrate. Figure 1 shows the sketch for the FIB nanomachining, and the material can be removed layer by layer using the high energetic bombarding ions from the focused ion beam. 


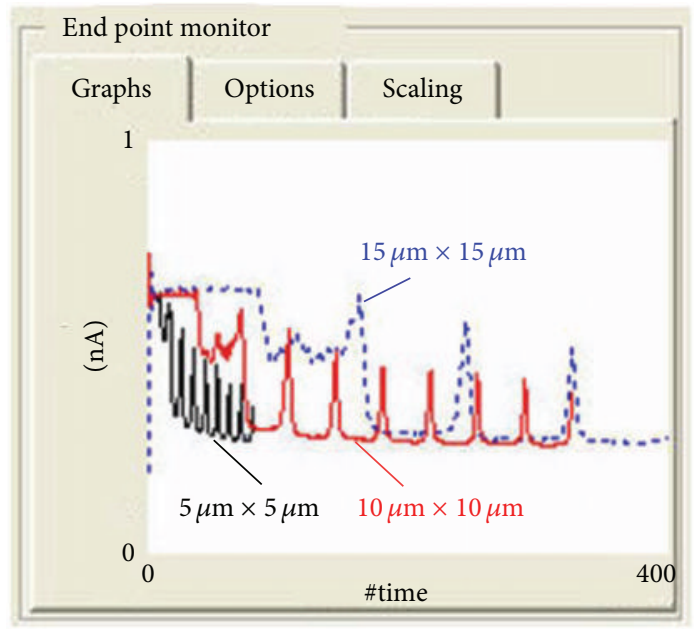

$$
\begin{aligned}
& --15 \mu \mathrm{m} \times 15 \mu \mathrm{m} \\
& -10 \mu \mathrm{m} \times 10 \mu \mathrm{m} \\
& -5 \mu \mathrm{m} \times 5 \mu \mathrm{m}
\end{aligned}
$$

(a)

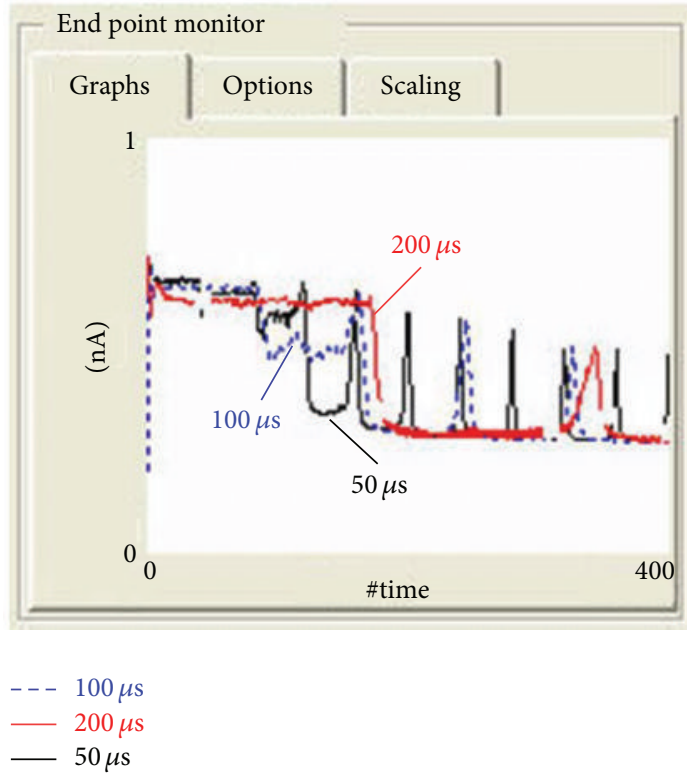

(b)

FiguRE 2: Sample-absorbed current versus machining time detected at different (a) milling area and (b) beam dwell time for the interface detection using the EPM.

As shown in Figure 2, the EPM in the FIB system, which collects the specimen-absorbed current as the EPD signal through a real-time monitor and an EPM plot (the sample inside the chamber of FIB system is connected to an electrometer amplifier to monitor the real-time current signal), was used to detect the sample-absorbed current signal for the interface detection assisted by the FIB cross sectioning. Using the EPD technique, the real-time patterning depth control for the plasmonic structure fabrication can be achieved via controlling machining time using the EPM. Table 1 gives the parameters used for the $\mathrm{Ag} / \mathrm{SiO}_{2}$ interface detection.

As shown in Table 1, the defined milling area for the EPM-assisted current signal detection should be relatively large if the machining efficiency is allowed, and $15 \mu \mathrm{m}$ by $15 \mu \mathrm{m}$ is selected for a better EPM control (Figure 2). Besides, a relatively high ion beam current (e.g., $0.3 \mathrm{nA}$ ) is used to obtain a strong detection signal and increase nanomachining efficiency. Accordingly, the machining time control has been achieved via interface detection using an ion beam current of $0.3 \mathrm{nA}$, and the ion doses used can be also obtained through the following:

$$
\begin{aligned}
\text { Ion dose } & =\text { Ion flux } * \text { Milling time, } \\
\text { Ion flux } & =6.24 \times 10^{18} * \frac{\text { Ion beam current }}{\text { Milling area }}, \\
\text { Milling area } & =\frac{\text { Defined frame area }}{\cos (\theta)},
\end{aligned}
$$

where $\theta$ is the angle between the normal line of the sample surface and the axis of the ion beam $\theta$ can alter when the sample stage tilts).
TABLE 1: Parameters used for $\mathrm{Ag} / \mathrm{SiO}_{2}$ interface detection.

\begin{tabular}{lcccc}
\hline $\begin{array}{l}\text { Milling area } \\
(\mu \mathrm{m} \times \mu \mathrm{m})\end{array}$ & $\begin{array}{c}\text { Dwell time } \\
(\mu \mathrm{s})\end{array}$ & $\begin{array}{c}\text { Current } \\
(\mathrm{nA})\end{array}$ & Overlap & $\begin{array}{c}\text { Ion flux } \\
\left(\text { ions } \mathrm{cm}^{-2} \mathrm{~s}^{-1}\right)\end{array}$ \\
\hline $15 \times 15$ & 100 & 0.3 & $50 \%$ & $8.32 \times 10^{14}$ \\
\hline
\end{tabular}

If patterning at or near an interface for a very thin film layer occurs, the ion beam current should be smaller to avoid seriously damaging the film layer surface or the adjacent area. Thus, in this work, the real-time sputtering depth control for the FIB-based actual nanopatterning can be achieved simply and conveniently just through calculation and transformation into sputtering time via selecting a small ion beam current (e.g., 10-100 pA) and calculating the required milling area given a normal incident angle of the ion beam (i.e., $\theta=0^{\circ}$ ) together with the other fixed milling parameters (e.g., overlap and dwell time). Consequently, a better control for the actual nanostructure fabrication can be achieved using this method assisted by the scanning electron microscopy (SEM) and atomic force microscopy (AFM) measurements.

\section{FIB-Assisted Interface Detection}

It is necessary to measure the thin film thickness and detect the interface between film layer and substrate in order to control patterning formation at/near the interface via the EPM control of the required milling time which may be calculated through the required ion dose. However, it is difficult to measure the film thickness by traditional methods (e.g., optical method based on thin film interference using fiber optics coupled microscope, AFM, ultrasonic pulse-echo technique of ultrasonic gages for measuring the thickness of 
TABLE 2: Material-dependent currents measured using the EPM for the same sample.

\begin{tabular}{|c|c|c|c|c|}
\hline \multirow{2}{*}{ Beam current (nA) } & \multirow{2}{*}{ Ion flux (ions $\mathrm{cm}^{-2} \mathrm{~s}^{-1}$ ) } & \multicolumn{3}{|c|}{ Material-dependent current (nA) } \\
\hline & & Ag oxide & Ag film & $\mathrm{SiO}_{2}$ substrate \\
\hline 0.3 & $8.32 \times 10^{14}$ & 0.76 & 0.70 & 0.3 \\
\hline 1.0 & $3.12 \times 10^{15}$ & 2.73 & 2.50 & 1.0 \\
\hline
\end{tabular}

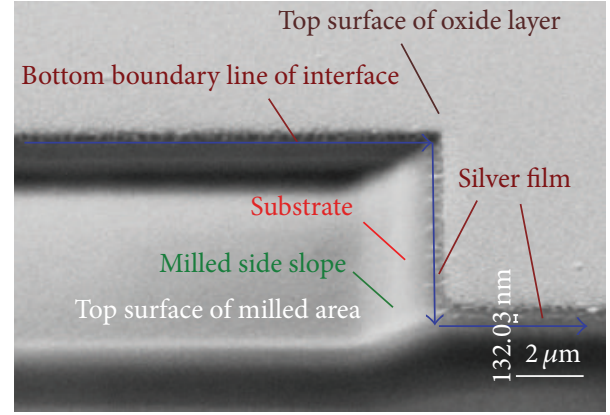

FIGURE 3: The $\mathrm{Ag} / \mathrm{SiO}_{2}$ interface cross section measured using SEM.

coatings on nonmetal substrates, etc.) if the deposited film is too thin (e.g., for the cases less than $100 \mathrm{~nm}$ thick). Thus, in this work, the EPM current curve was used as the end point detection signal (i.e., the abrupt transition point of the detected signal curve for the sample-absorbed current versus the milling time during the milling process) to obtain the required ion dose and milling time for the interface detection. The operation procedures related to FIB cross sectioning include rectangle milling, regular cross section, cleaning cross section, and measurement for thin film thickness via SEM imaging for the interface cross section.

Figure 3 shows the Ag/substrate interface cross section detected using the FIB/SEM dual beam system. As shown in Figure 3, the sidewalls of the milled zones are approximately $\mathrm{V}$-shaped due to the Gaussian beam and the redeposition of the sputtered material debris on the sidewalls (the V-shape sidewall formation after FIB milling can be also verified in Figure 4), and the thickness of the Ag film deposited on the glass substrate is around $132 \mathrm{~nm}$.

As shown in Figure 5, there are 9 raster passes in all: the first pass is for sputtering the oxide layer and silver film (see Figure 5(a)); the second pass is for milling transition from Ag film to $\mathrm{SiO}_{2}$ substrate (see Figure 5(b)); the left passes, as shown in Figure 5(c), are used to mill deep into $\mathrm{SiO}_{2}$ substrate. Time used for sputtering oxide layer is $3 \mathrm{~s}$. Milling stops in the deposited film material from $3 \mathrm{~s}$ to $85 \mathrm{~s}$; at $85 \mathrm{~s}$ (ion dose used is $7.07 \times 10^{16}$ ions $\mathrm{cm}^{-2}$ ), milling just occurs at the $\mathrm{Ag} / \mathrm{SiO}_{2}$ interface boundary, that is, just starting to sputter the substrate material; subsequently, milling occurs in both $\mathrm{Ag}$ layer and $\mathrm{SiO}_{2}$ substrate until $163 \mathrm{~s}$, which is a transition process from film machining to substrate machining. Almost all film material is removed at $163 \mathrm{~s}$ when a relatively clean interface (a clean plane interface could be theoretically achieved at this moment if under ideal conditions; however, in this case, the film layer subjected to ion beam irradiation was not absolutely removed evenly, resulting in some nanoscale residues at the
$\mathrm{Ag} / \mathrm{SiO}_{2}$ interface) between the film layer and the substrate is obtained (Figure 6). Afterwards, as shown in Figure 5, milling continues to occur deep into the substrate until $745 \mathrm{~s}$ (ion dose used is $6.20 \times 10^{17}$ ions $\mathrm{cm}^{-2}$ ).

As shown in Figure 6, the deposited film material was not completely sputtered at the "exact" interface; that is, some silver residues may remain at the interface (it should be indicated that the reported EPD accuracy is in the range of a few tens of nanometers which results from the complex interfacial mixing with the ion bombardment depending on beam energy, material properties, crystallographic orientation, substrate surface roughness, and quality of deposited thin films, etc. $[23,24])$. Thus, the machining end point could be normally below the "exact" interface to completely sputter the deposited film material. As a result, the sampleabsorbed current drops due to dielectric substrate machining (see Figure 5(c)).

\section{Material-Dependent Currents Measured during Interface Detection}

Material-dependent currents in Table 2 are measured via the EPM signal curves. The detected substrate-absorbed current is equal to the ion beam current used, and the strength of sample-absorbed current signal increases with the ion flux. Under the same machining conditions, compared with the Ag-absorbed current signal, the $\mathrm{Ag}_{2} \mathrm{O}$-absorbed current signal is the strongest and the $\mathrm{SiO}_{2}$-absorbed current signal is the weakest, resulting from different material-dependent average atomic numbers (material with higher average atomic number can emit more secondary ions/electrons; the atomic numbers for $\mathrm{Ag}_{2} \mathrm{O}, \mathrm{Ag}$, and $\mathrm{SiO}_{2}$ are 232, 108, and 60, resp.), material conductivity, and material sputtering yield for the secondary ions (sputtering yield $Y=$ ejected atom number/incident ion number, i.e., $Y=\rho d / \sigma$, where $\rho$ is the density (atoms $\mathrm{cm}^{-3}$ ), $d$ is the sputtering depth $(\mathrm{cm})$, and $\sigma$ is the ion dose (ions $\mathrm{cm}^{-2}$ )).

The detected current signal ratios for $I_{\text {Oxide }} / I_{\text {Film }}$ and $I_{\text {Substrate }} / I_{\text {Film }}$ are approximately constant (Table 3 ) despite encountering significant changes of ion fluxes and ion beam currents, which actually indicates that the materialdependent current signal ratio detected via the EPM is independent of processing conditions.

The quantitative correlation between the sampleabsorbed current $I_{S}$ and the ion beam current $I_{B}$ can be approximately expressed as a linear function for the same material which is given as

$$
I_{S}=\left(1+K_{E}-K_{I}\right) \times I_{B}=K \times I_{B}
$$




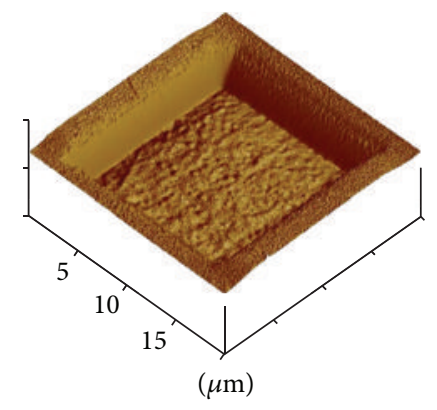

$x 5.000 \mu \mathrm{m} / \mathrm{div}$

$z 500.000 \mathrm{~nm} / \mathrm{div}$

(a)

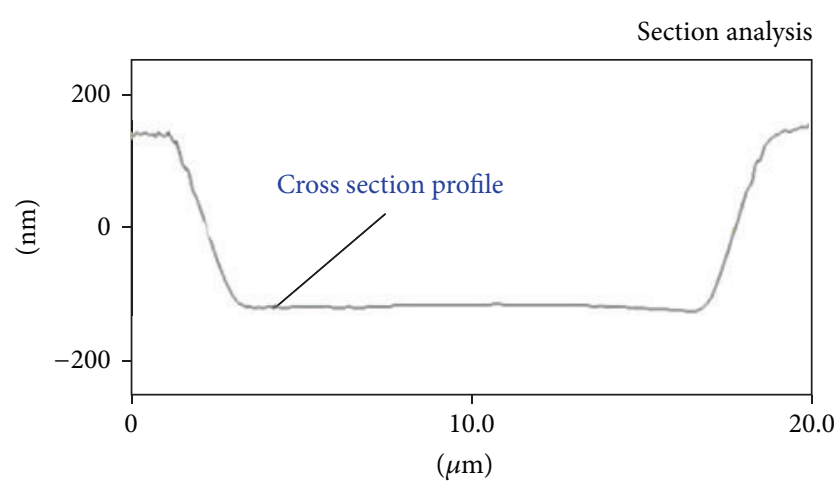

(b)

FIgURE 4: (a) 3D view and (b) 2D cross section analysis for the FIB-induced V-shape sidewall measured using AFM.

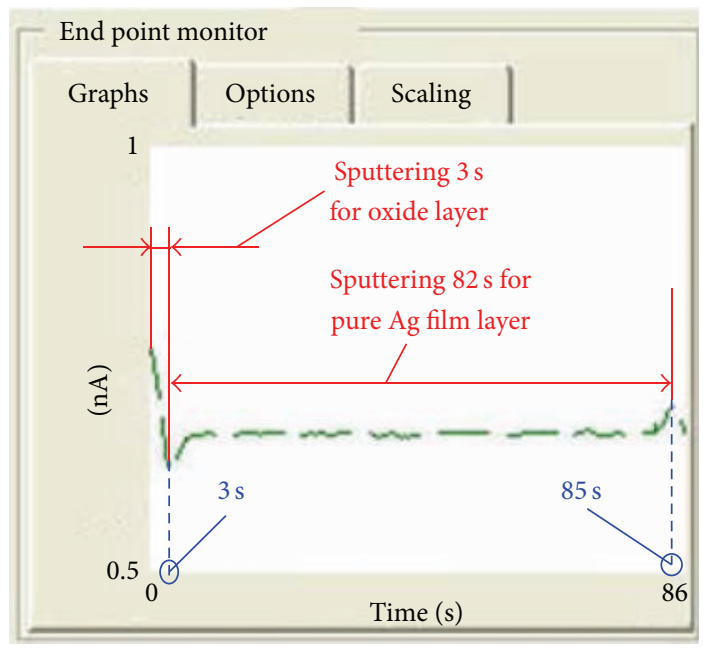

(a)

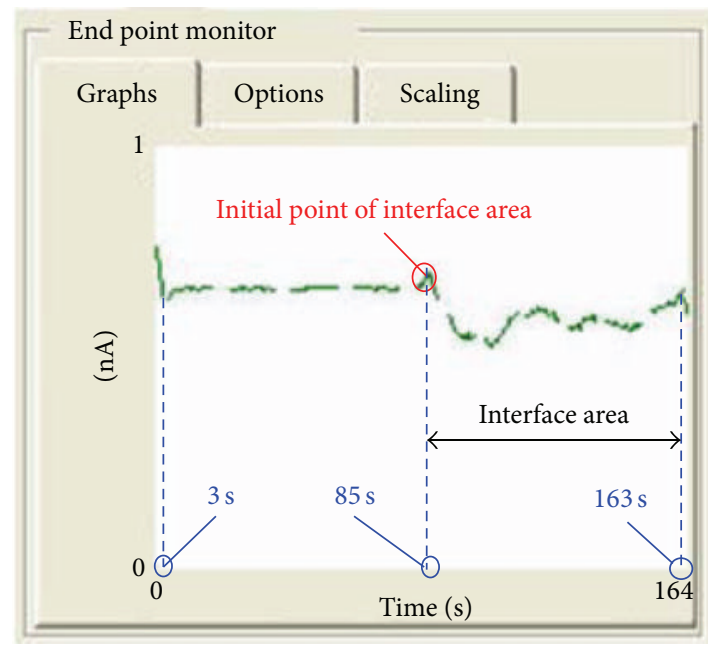

(b)

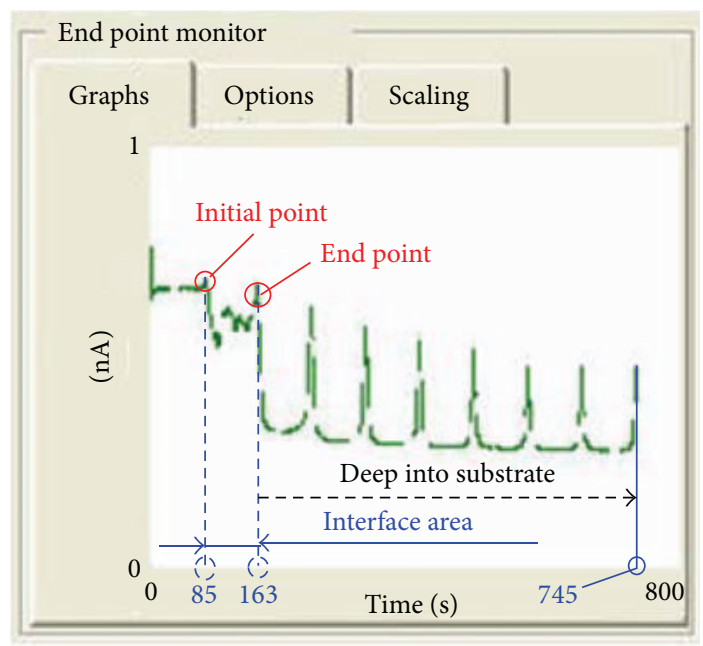

(c)

Figure 5: Patterning depth control via controlling machining time using the $\mathrm{EPM}$ for sputtering the (a) oxide layer and $\mathrm{Ag}$ film, (b) $\mathrm{Ag} / \mathrm{SiO}{ }_{2}$ interface, and (c) $\mathrm{SiO}_{2}$ substrate. 


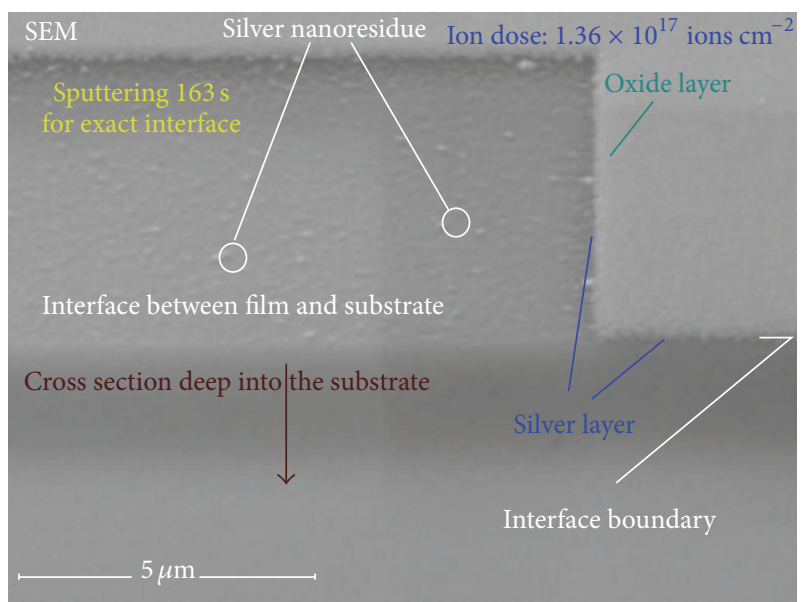

FIGURE 6: The interface detected using FIB/SEM dual beam system after FIB milling $163 \mathrm{~s}$.

TABLE 3: Material-dependent current ratios detected for the same sample.

\begin{tabular}{|c|c|c|}
\hline \multirow{2}{*}{ Ion flux (ions $\mathrm{cm}^{-2} \mathrm{~s}^{-1}$ ) } & \multicolumn{2}{|c|}{ Material-dependent current signal ratio } \\
\hline & Oxide/film & Substrate/film \\
\hline $8.32 \times 10^{14}$ & 1.09 & 0.43 \\
\hline $3.12 \times 10^{15}$ & 1.09 & 0.40 \\
\hline
\end{tabular}

TABLE 4: Linear coefficients for sample-absorbed current and ion beam current.

\begin{tabular}{lccc}
\hline Ion flux (ions $\mathrm{cm}^{-2} \mathrm{~s}^{-1}$ ) & \multicolumn{3}{c}{$K$} \\
& $\mathrm{Ag}$ oxide & $\mathrm{Ag} \mathrm{film}$ & $\mathrm{SiO}_{2}$ substrate \\
\hline $8.32 \times 10^{14}$ & 2.53 & 2.33 & 1.0 \\
$3.12 \times 10^{15}$ & 2.73 & 2.50 & 1.0 \\
\hline
\end{tabular}

TABLE 5: Linear coefficients for sample-absorbed current and ion beam current.

\begin{tabular}{lccc}
\hline Sample materials & Ag oxide & Ag film & $\mathrm{SiO}_{2}$ substrate \\
\hline Average value of $K$ & 2.63 & 2.42 & 1.0 \\
\hline
\end{tabular}

where $K_{E}$ and $K_{I}$ denote the coefficients for electrons and ions, respectively, and $K=1+K_{E}-K_{I}$.

The values of $K$ for Ag oxide, $\mathrm{Ag}$ film, and $\mathrm{SiO}_{2}$ substrate can be determined through the detected sample-absorbed currents (Tables 2 and 4). Table 5 lists the average values of $K$ for $\mathrm{Ag}$ oxide, $\mathrm{Ag}$ film, and $\mathrm{SiO}_{2}$ substrate, which is achieved through the data in Table 4. Thus, for the case of FIB milling the quartz substrate with the deposited Ag film, the quantitative expressions for the sample-absorbed currents and ion beam current can be given as

$$
\begin{aligned}
I_{\mathrm{Ag}_{2} \mathrm{O}} & =2.63 \times I_{B}, \\
I_{\mathrm{Ag}} & =2.42 \times I_{B}, \\
I_{\mathrm{SiO}_{2}} & =I_{B} .
\end{aligned}
$$

\section{Plasmonic Nanostructure Development Based on Interface Detection}

The successful detection for the interface between coated metal and dielectric substrate is important to the next step to fabricate functional plasmonic structures, and the real-time patterning depth control of functional plasmonic structure fabrication using relatively small ion beam currents has also been achieved through controlling milling time when the ion dose for fabricating the plasmonic structure is obtained after the interface detection; that is, through interface detection for the Ag-deposited substrate with a multilayer structure, the end point detection technique can be further employed to achieve the real-time control of the patterning depth via controlling the ion dose or milling time during the process of plasmonic structure fabrication. Accordingly, the patterning geometries and dimensions can be changed after determining the ion dose required for patterning at or near the interface via interface detection for the Ag-coated substrate with a multilayer structure based on (1).

As shown in Figures 7 and 8, some Ag-coated plasmonic nanostructures have been fabricated and measured using the FIB/SEM system and AFM based on the achieved methodology of the interface detection for FIB nanofabrication of the plasmonic structures, including nanoscale pentagram hole/particle arrays and square nanohole arrays. The primary concern for FIB nanofabrication of these plasmonic structures is the precise dimension and patterning control for fabricating the nanoscale hole/particle arrays; for example, the concern for angle fabrication requires high machining resolution and small beam spot diameter, which requires relatively small ion beam currents (e.g., 10-50 pA) although the fabrication efficiency is low. In addition, the AFM-measured depth of the fabricated nanoholes is around $135 \mathrm{~nm}$ (Figure 7), which nearly agrees with the film thickness $132 \mathrm{~nm}$ measured through the interface detection (Figure 3), demonstrating the feasibility and reliability of the achieved methodology for the real-time precise patterning depth control during the plasmonic nanostructure fabrication process based on the FIB-assisted interface detection.

The developed nanoscale hole/particle arrays can find potential plasmonic device applications (e.g., biosensors) through detecting the LSPR signals of the optical field intensity distribution, optical spectra, and/or refractive indices for the analytes via transmission and/or reflection mode sensing $[32,36-42]$. It is reported that the plasmonic pentagram nanoholes can significantly enhance transmission and Efield, and the plasmonic pentagram nanohole array can enhance the LSPR. Thus, the plasmonic pentagram nanoholes have potential applications in the fields of imaging, focusing, and biosensing [41].

Figure 9 is a schematic example to illustrate the application for transmission mode sensing using a plasmonic nanohole array, where the transmitted optical field intensity for the analytes can be detected by the scanning near-field optical microscope (SNOM) tip for the sensing signal. The transmission (reflection) mode means that the light illuminates the plasmonic structure from the substrate (analyte) side, and the detection is at the analyte side. 

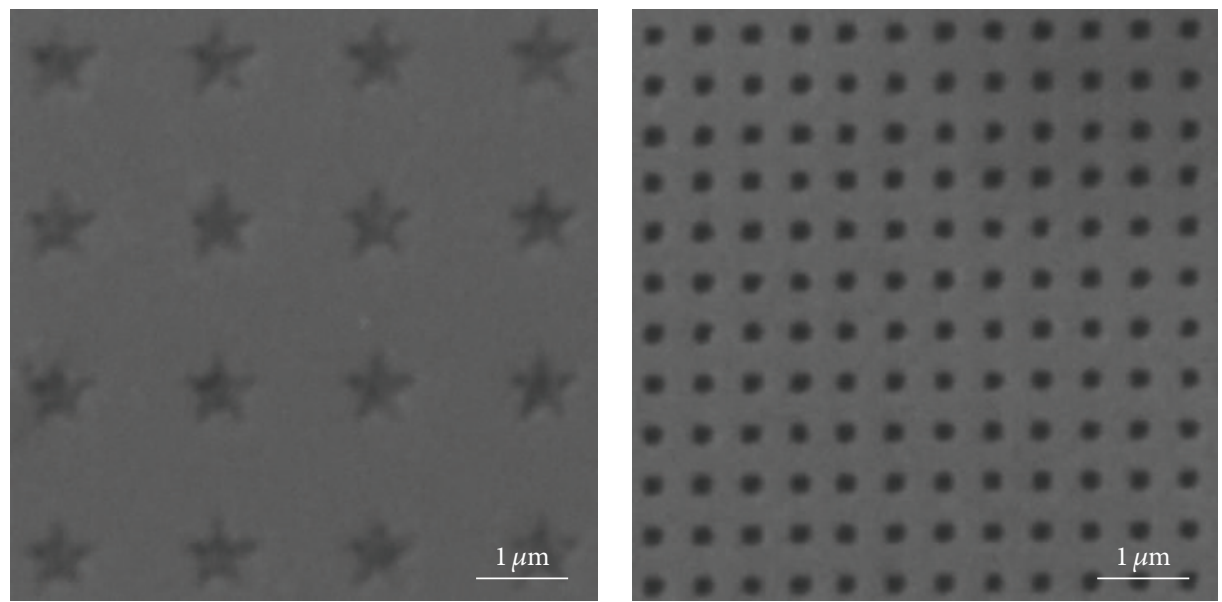

(a)
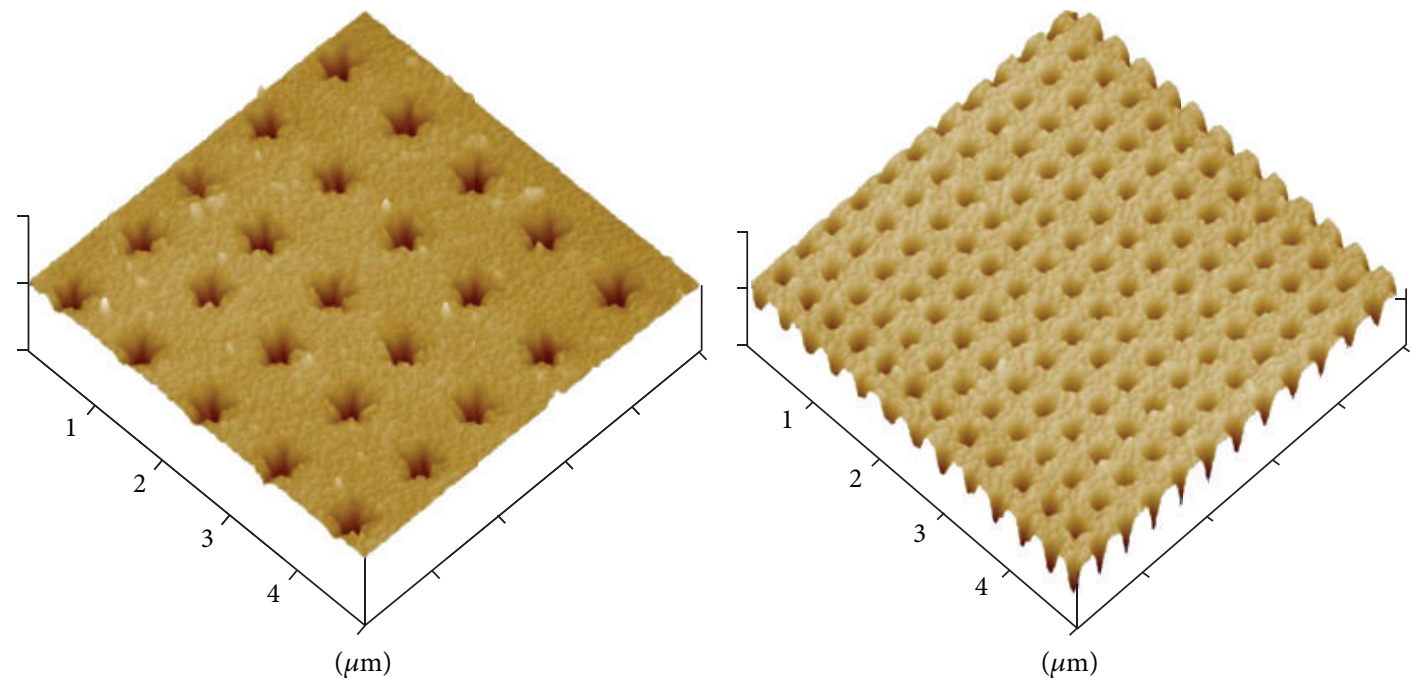

$x 1.000 \mu \mathrm{m} / \mathrm{div}$

$x 1.000 \mu \mathrm{m} / \mathrm{div}$

$z \quad 100.000 \mathrm{~nm} / \mathrm{div}$

$z 100.000 \mathrm{~nm} / \mathrm{div}$

(b)
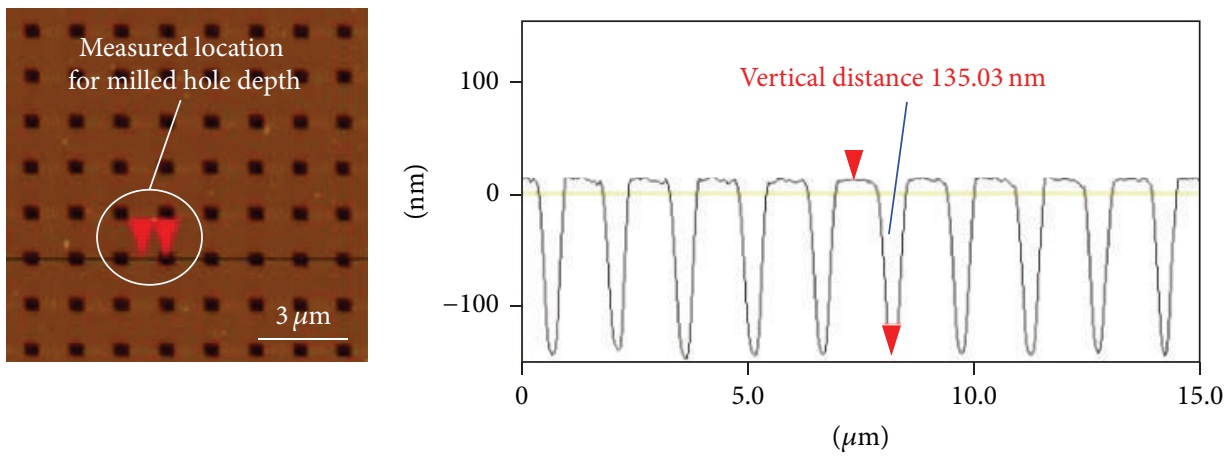

(c)

FIGURE 7: FIB-fabricated pentagram and square plasmonic nanohole arrays measured using the (a) SEM and ((b)-(c)) AFM.

\section{Conclusion}

The FIB-assisted interface detection was proposed and successfully carried out using the sample-absorbed current as the detection signal, and the patterning depth control for the plasmonic structure fabrication was achieved through controlling machining time or ion dose using the EPM. Materialdependent currents were measured through the EPM signal 


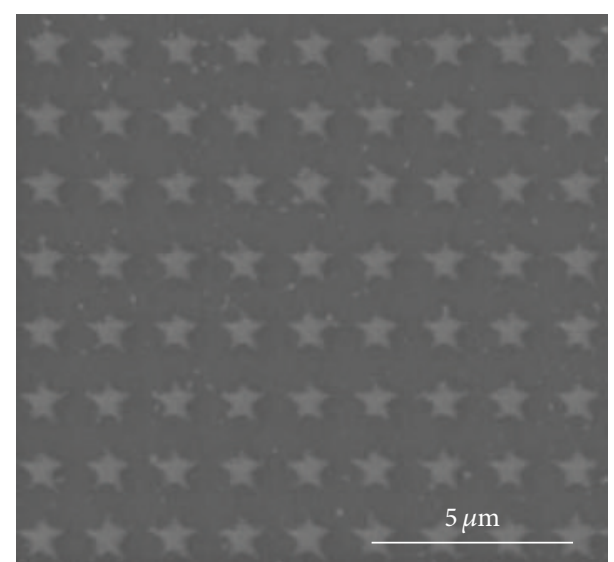

FIGURE 8: FIB-fabricated pentagram plasmonic nanostar array measured using the SEM.

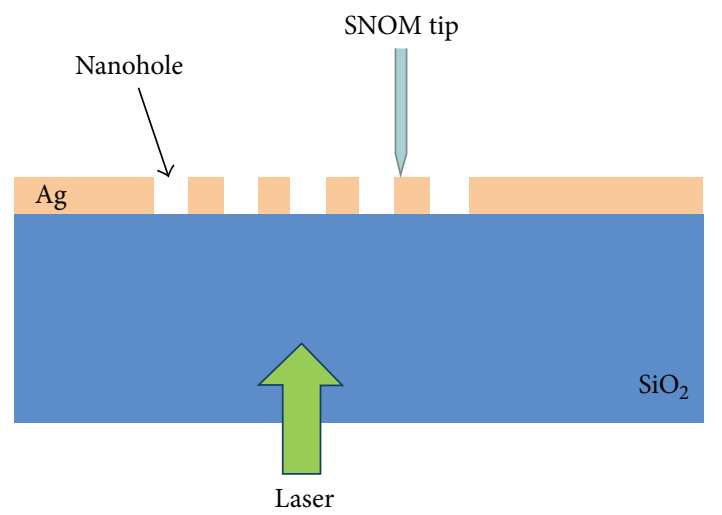

FIGURE 9: Sketch for illustrating the principle of transmission mode sensing based on a plasmonic nanohole array.

curves, and the quantitative models for the sample-absorbed currents and the ion beam current were also developed. The functional plasmonic nanostructures (pentagram nanoscale hole/particle arrays and square nanohole arrays for the potential LSPR biosensing applications) were fabricated and measured based on the achieved systematic methodology of the interface detection for FIB nanofabrication of patterned nanostructures for potential plasmonic device applications (e.g., LSPR biosensors). It was shown that the proposed methodology on the FIB-assisted interface detection can be conveniently used for the real-time control and precise fabrication of different functional plasmonic nanostructures with different geometries and dimensions.

\section{Conflict of Interests}

The authors declare that there is no conflict of interests regarding the publication of this paper.

\section{Acknowledgments}

The work is supported by the Research Foundation for Advanced Talents of Jiangsu University under Grant no.
14JDG020, China, and the A STAR (Agency for Science, Technology and Research) under SERC Grant no. 072101 0023, Singapore.

\section{References}

[1] Z. Yu, H. Gao, W. Wu, H. Ge, and S. Y. Chou, "Fabrication of large area subwavelength antireflection structures on Si using trilayer resist nanoimprint lithography and liftoff," Journal of Vacuum Science and Technology B, vol. 21, no. 6, p. 2874, 2003.

[2] G. Kostovski, D. J. White, A. Mitchell, and P. R. Stoddart, "Nanoimprinting on optical fiber end faces for chemical sensing," in 19th International Conference on Optical Fibre Sensors, vol. 7004 of Proceedings of SPIE, April 2008.

[3] S. A. Boden and D. M. Bagnall, "Tunable reflection minima of nanostructured antireflective surfaces," Applied Physics Letters, vol. 93, no. 13, Article ID 133108, 2008.

[4] Y. Lin, Y. Zou, Y. Mo, J. Guo, and R. G. Lindquist, "E-beam patterned gold nanodot arrays on optical fiber tips for localized surface plasmon resonance biochemical sensing," Sensors, vol. 10, no. 10, pp. 9397-9406, 2010.

[5] Y. Lin, Y. Zou, and R. G. Lindquist, "A reflection-based localized surface plasmon resonance fiber-optic probe for biochemical sensing," Biomedical Optics Express, vol. 2, no. 3, pp. 478-484, 2011.

[6] N. Chekurov, K. Grigoras, A. Peltonen, S. Franssila, and I. Tittonen, "The fabrication of silicon nanostructures by local gallium implantation and cryogenic deep reactive ion etching," Nanotechnology, vol. 20, no. 6, Article ID 065307, 2009.

[7] Y. F. Chang, Q. R. Chou, I. Y. Lin, and C. H. Lee, "Fabrication of high-aspect-ratio silicon nanopillar arrays with the conventional reactive ion etching technique," Applied Physics A, vol. 86, pp. 193-196, 2007.

[8] S. H. G. Teo, A. Q. Liu, G. L. Sia, C. Lu, J. Singh, and M. B. Yu, "Deep reactive ion etching for pillar type nanophotonic crystal," International Journal of Nanoscience, vol. 4, no. 4, pp. 567-574, 2005.

[9] N. A. Kouklin and J. Liang, "Ultradense GaN nanopillar and nanopore arrays by self-assembly nanopatterning," Journal of Electronic Materials, vol. 35, no. 5, pp. 1133-1137, 2006.

[10] S. W. Kim, "High-temperature fiber optic sensor using a grating on an angled fiber tip," Japanese Journal of Applied Physics, vol. 41, no. 3, p. 1431, 2002.

[11] X. Ma, H. Huo, W. Wang et al., "Surface-enhanced Raman scattering sensor on an optical fiber probe fabricated with a femtosecond laser," Sensors, vol. 10, no. 12, pp. 11064-11071, 2010.

[12] J. W. Menezes, J. Ferreira, M. J. L. Santos, L. Cescato, and A. G. Brolo, "Large-area fabrication of periodic arrays of nanoholes in metal films and their application in biosensing and plasmonicenhanced photovoltaics," Advanced Functional Materials, vol. 20, no. 22, pp. 3918-3924, 2010.

[13] S. E. Wu, C. P. Liu, T. H. Hsueh, H. C. Chung, C. C. Wang, and C. Y. Wang, "Anomalous formation of InGaN/GaN multiplequantum-well nanopillar arrays by focused ion beam milling," Nanotechnology, vol. 18, no. 44, Article ID 445301, 2007.

[14] I. Utke, P. Hoffmann, and J. Melngailis, "Gas-assisted focused electron beam and ion beam processing and fabrication," Journal of Vacuum Science and Technology B, vol. 26, no. 4, pp. 1197-1276, 2008.

[15] M. Kolíbal, T. Matlocha, T. Vystavěl, and T. Šikola, "Low energy focused ion beam milling of silicon and germanium 
nanostructures," Nanotechnology, vol. 22, no. 10, Article ID 105304, 2011.

[16] H. X. Wang, W. Zhou, Y. Cui, G. H. Wang, and P. P. Shum, "Focused ion beam nanoscale patterned transmissionenhanced fiber-optic tips," Journal of Nanoscience and Nanotechnology, vol. 13, no. 7, pp. 4581-4586, 2013.

[17] H. X. Wang and W. Zhou, "Focused ion beam nanomachining for analyzing effects of light-structure interaction on hair color," Journal of Biomaterials and Tissue Engineering, vol. 4, no. 5, pp. 411-415, 2014.

[18] W. Zhou, H. X. Qian, and L. M. Wang, "Maskless fabrication of highly-ordered periodic nanopillars using FIB and Bitmap control," Microscopy and Microanalysis, vol. 11, supplement 2, pp. 822-823, 2005.

[19] S. Zhu and W. Zhou, "Optical properties and immunoassay applications of noble metal nanoparticles," Journal of Nanomaterials, vol. 2010, Article ID 562035, 12 pages, 2010.

[20] O. Kvítek, J. Siegel, V. Hnatowicz, and V. Švorčík, "Noble metal nanostructures influence of structure and environment on their optical properties," Journal of Nanomaterials, vol. 2013, Article ID 743684, 15 pages, 2013.

[21] M. G. Sreenivasan, S. Malik, S. Thigulla, and B. R. Mehta, "Dependence of plasmonic properties of silver island films on nanoparticle size and substrate coverage," Journal of Nanomaterials, vol. 2013, Article ID 247045, 8 pages, 2013.

[22] T. Zhang and F. Shan, "Development and application of surface plasmon polaritons on optical amplification," Journal of Nanomaterials, vol. 2014, Article ID 495381, 16 pages, 2014.

[23] H. X. Qian, W. Zhou, X. M. Li, J. M. Miao, and L. E. N. Lim, "Accurate detection of interface between $\mathrm{SiO}_{2}$ film and $\mathrm{Si}$ substrate," Applied Surface Science, vol. 253, no. 12, pp. 5511-5515, 2007.

[24] S. T. Davies and B. Khamsehpour, "End-point detection using absorbed current, secondary electron, and secondary ion signals during milling of multilayer structures by focused ion beam," Journal of Vacuum Science \& Technology B, vol. 11, no. 2, pp. 263-267, 1993.

[25] Y. Z. Huang and D. Cockayne, "Control of nanostructures induced by ion sputtering," Nanotechnology, vol. 18, no. 2, Article ID 025305, 2007.

[26] R. Hill, J. C. Morgan, R. G. Lee, and T. Olson, "FIB endpoint detection and depth resolution," Microelectronic Engineering, vol. 21, no. 1-4, pp. 201-204, 1993.

[27] H. X. Qian and W. Zhou, "Focused ion beam irradiation of $\mathrm{ZnO}$ film: an atomic force microscopy study, Journal of Physics D: Applied Physics, vol. 42, no. 10, Article ID 105304, 2009.

[28] B. Khamsehpour and S. T. Davies, "Micromachining of semiconductor materials by focused ion beams," Vacuum, vol. 45, no. 12, pp. 1169-1173, 1994.

[29] L. R. Harriott, A. Wagner, and F. Fritz, "Integrated circuit repair using focused ion beam milling," Journal of Vacuum Science \& Technology B, vol. 4, p. 181, 1986.

[30] P. J. Heard, J. R. A. Cleaver, and H. Ahmed, "Application of a focused ion beam system to defect repair of VLSI masks," Journal of Vacuum Science and Technology B, vol. 3, no. 1, p. 87, 1985.

[31] S. L. Zhu, W. Zhou, G.-H. Park, and E. P. Li, "Numerical design methods of nanostructure array for nanobiosensing," Plasmonics, vol. 5, no. 3, pp. 267-271, 2010.

[32] L. Wu, P. Bai, X. Zhou, and E. P. Li, "Reflection and transmission modes in nanohole-array-based plasmonic sensors," IEEE Photonics Journal, vol. 4, no. 1, pp. 26-33, 2012.
[33] L. Pang, G. M. Hwang, B. Slutsky, and Y. Fainman, "Spectral sensitivity of two-dimensional nanohole array surface plasmon polariton resonance sensor," Applied Physics Letters, vol. 91, no. 12, Article ID 123112, 2007.

[34] T. Chung, S.-Y. Lee, E. Y. Song, H. Chun, and B. Lee, "Plasmonic nanostructures for nano-scale bio-sensing," Sensors, vol. 11, no. 11, pp. 10907-10929, 2011.

[35] A.-P. Blanchard-Dionne, L. Guyot, S. Patskovsky, R. Gordon, and M. Meunier, "Intensity based surface plasmon resonance sensor using a nanohole rectangular array," Optics Express, vol. 19, no. 16, pp. 15041-15046, 2011.

[36] G. Si, A. J. Danner, S. L. Teo, E. J. Teo, J. Teng, and A. A. Bettiol, "Photonic crystal structures with ultrahigh aspect ratio in lithium niobate fabricated by focused ion beam milling," Journal of Vacuum Science \& Technology B: Microelectronics and Nanometer Structures, vol. 29, no. 2, Article ID 021205, 2011.

[37] R. M. Langford, P. M. Nellen, J. Gierak, and Y. Fu, "Focused ion beam micro- and nanoengineering," MRS Bulletin, vol. 32, no. 5, pp. 417-423, 2007.

[38] Z. L. Wang, Q. Wang, H. J. Li et al., “The field emission properties of high aspect ratio diamond nanocone arrays fabricated by focused ion beam milling," Science and Technology of Advanced Materials, vol. 6, no. 7, pp. 799-803, 2005.

[39] O. Wilhelmi, S. Reyntjens, C. Mitterbauer, L. Roussel, D. J. Stokes, and D. H. W. Hubert, "Rapid prototyping of nanostructured materials with a focused ion beam," Japanese Journal of Applied Physics, vol. 47, no. 6, pp. 5010-5014, 2008.

[40] G. H. Wang, X. T. Zheng, P. P. Shum, C. M. Li, H. P. Ho, and L. $\mathrm{M}$. Tong, "Live cell index sensing based on the reflection mode of tilted fiber tip with gold nanoparticles," in Proceedings of the 9th International Conference on Optical Communications and Networks, vol. 574CP, p. 116, Nanjing, China, 2010.

[41] S. Zhu and W. Zhou, "Plasmonic properties of two-dimensional metallic nanoholes fabricated by focused ion beam lithography," Journal of Nanoparticle Research, vol. 14, article 652, 2012.

[42] S. L. Zhu and W. Zhou, "Optical properties of pentagram nanostructures based on localized surface plasmon resonance," Journal of Optics, vol. 40, no. 2, pp. 65-70, 2011. 

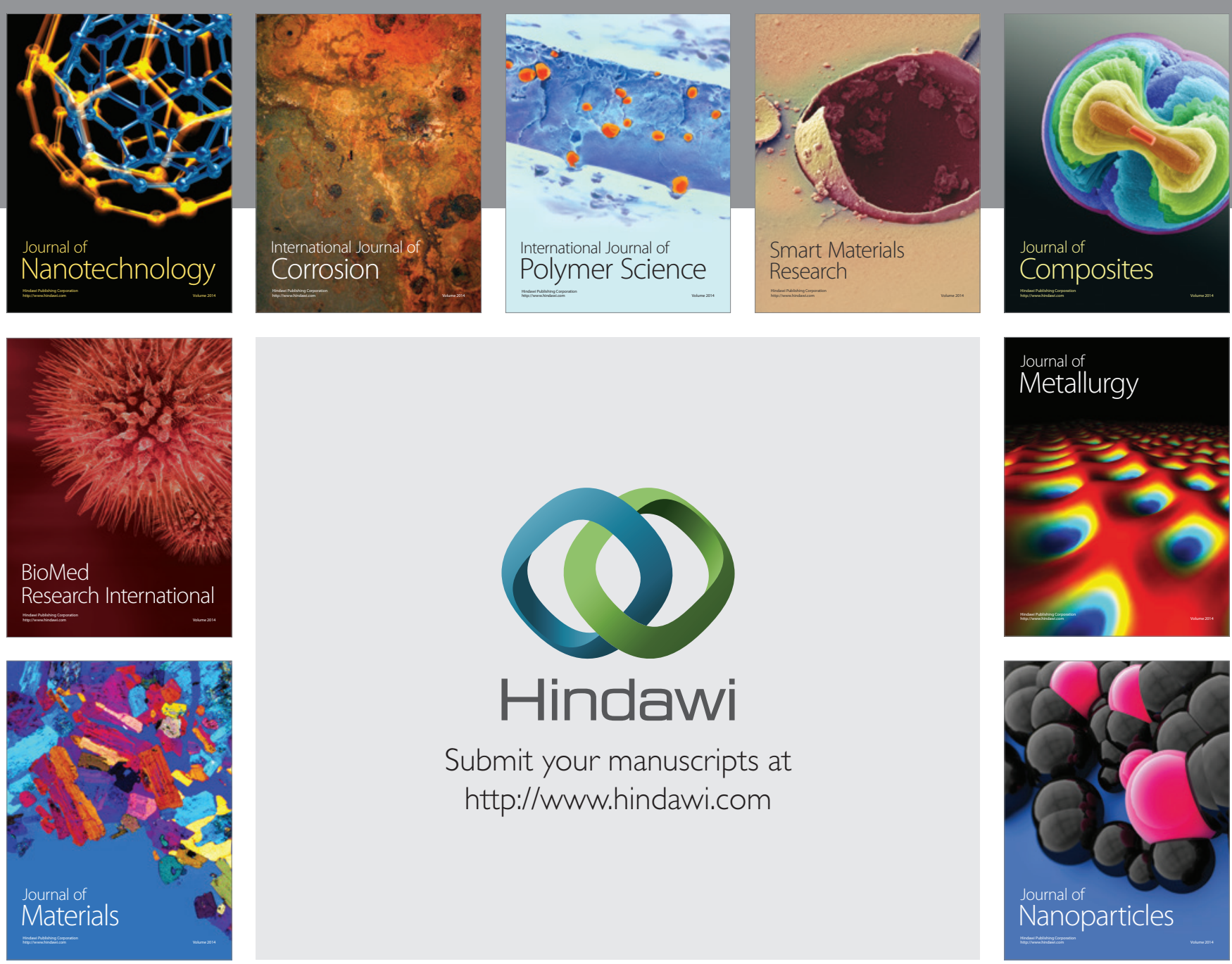

Submit your manuscripts at http://www.hindawi.com
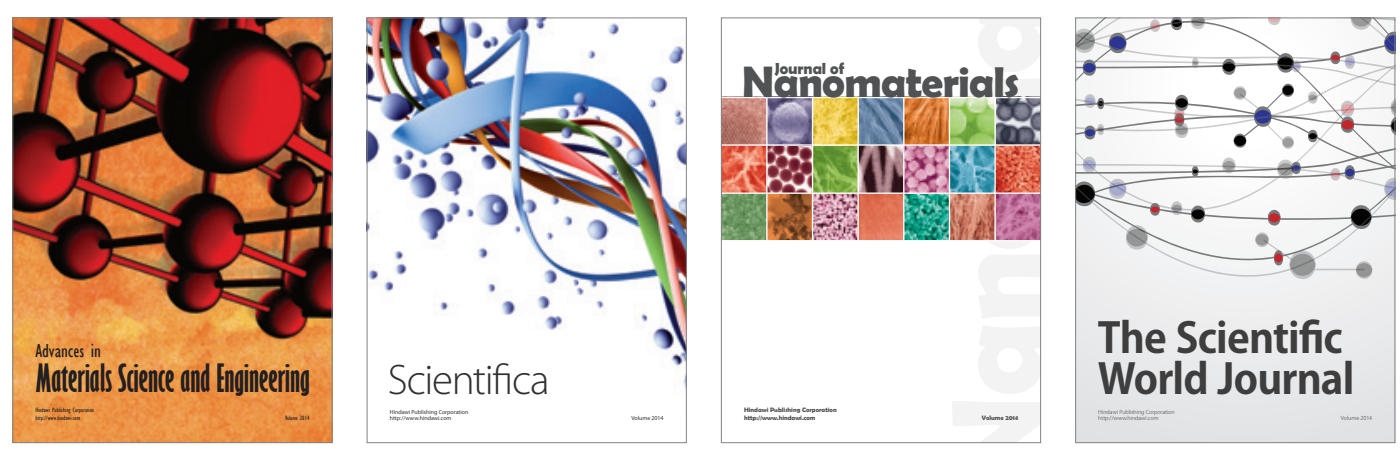

\section{The Scientific World Journal}
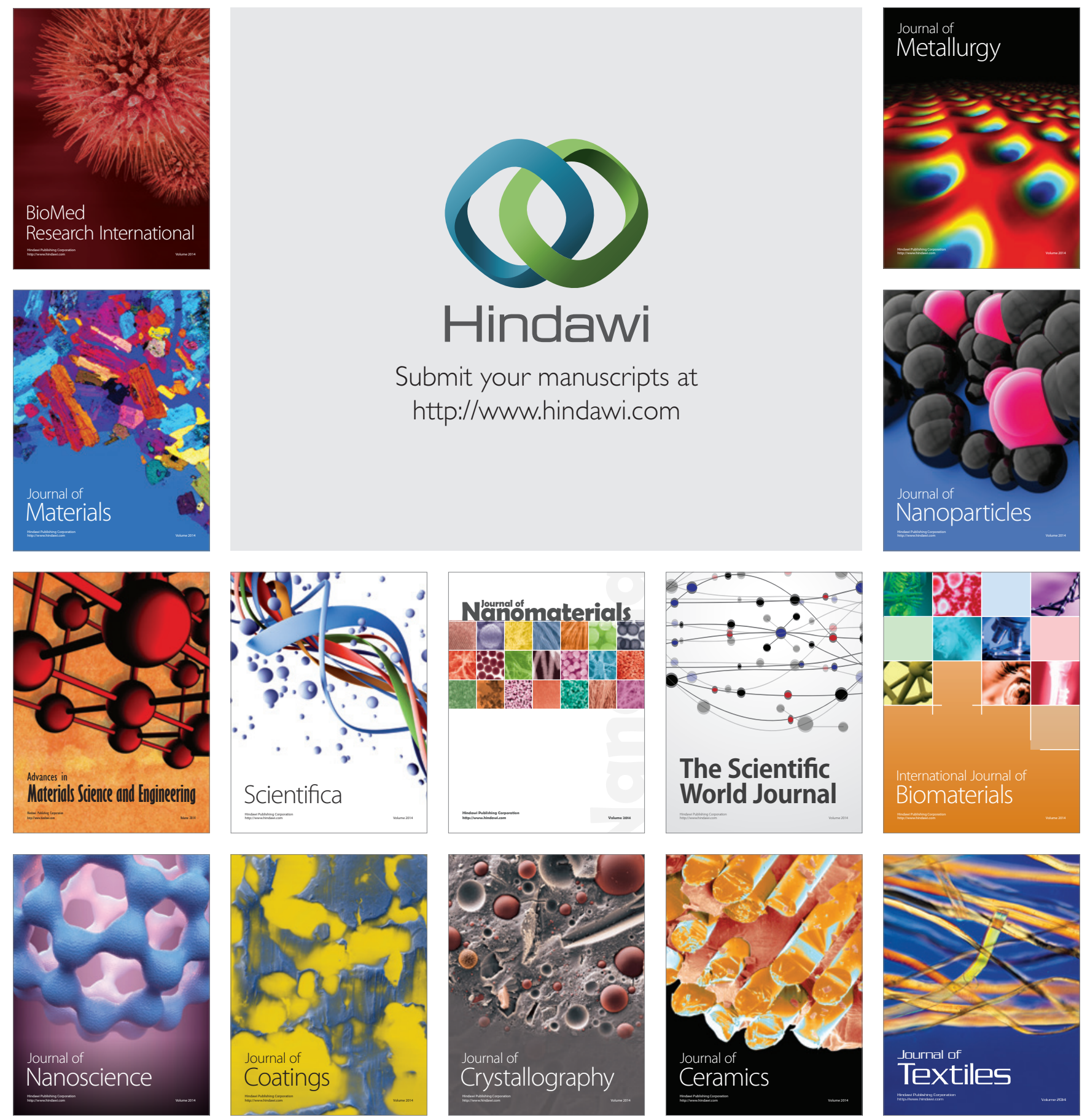